\title{
Numerical Modeling of Specimen Geometry for Quantitative Multiple Detector EDS
}

\author{
Weizong $\mathrm{Xu}^{1}$, J. Houston Dycus ${ }^{1}$ and James M. LeBeau ${ }^{1}$ \\ 1. Department of Materials Science and Engineering, North Carolina State University, Raleigh, NC \\ 27695, USA
}

By combining multiple X-ray detectors to enlarge the total detector area, new energy dispersive X-ray spectrometers exhibit dramatically enhanced collection efficiency. This provides significantly higher signal-to-noise ratio, enabling elemental mapping at the atomic scale [1-4]. The increased complexity in multi-detector systems has also brought new challenges in terms of absorption correction and X-rays being shadowed by holder as a function of tilt. To this end, we have previously developed a numerical that combines detector geometry, holder shadowing, Be filtering, and specimen absorption to quantitatively analyze elemental quantification on an atom-column by atom-column basis [3,5]. The numerical model incorporates detector and holder geometries by angularly discretizing the collected Xrays [5]. The effective detector solid angle, $\Omega_{\text {eff }}$ is determined by integrating over all discretized X-rays, $i$, collected from every sample slice along the depth direction, $t$, and then can be further used to quantify absolute counts and counts ratio of the sample.

$$
\Omega_{\text {eff }}=\frac{1}{N} \sum_{t} \sum_{i} B_{i, \text { frane }} B_{i, \text { clip }} B_{i, \text { grid }} A_{i, \text { spec }}(t) A_{i, \text { carr }} d \theta d \varphi \sin \theta_{i}
$$

where $\theta$ and $\varphi$ are the take-off and azimuth angle of discretized X-rays, respectively. $A_{i, s p e c}$ and $A_{i, \text { car }}$ represents the X-ray absorption factors from Be specimen carrier, respectively. In addition, the specimen holder frame $B_{i, f r a m e}$, sample securing clip $B_{i, \text { clip }}$ and/or supporting grid $B_{i, \text { grid }}$ can absorb nearly $100 \%$, i.e., $\sim$ block, of the X-rays.

In this talk, we build on the numerical approach to accurately calculate specimen absorption due to distorted specimen geometry commonly seen at the edge of TEM samples. We will explore the impact of specimen geometry using a 3D model built from bright-field TEM and cross-sectional SEM observations. We will show how the specimen object can be segmented in three-dimension using a tetrahedral mesh utilizing algorithms developed for finite element modeling (Figure 1a). The X-ray path from each depth of the specimen to its exit surface, $1_{i, t}$, can then be calculated via calculating the intersection of the beam path with the meshed tetrahedra. Figure $1 \mathrm{~b}$ provides an illustration of one such beam path and its intersections through the specimen (black tetrahedra). The quality $l_{i, t}$ then can be incorporated into the Equation (1) as the sample absorption term $A_{i, s p e c}$,

$$
A_{i, s p e c}(t)=\exp \left(-l_{i, t} \mu \rho\right)
$$

We will show that by employing the numerical model the asymmetric behavior of X-ray counts and ratios can be correctly predicted as a function of specimen tilt as seen in Figure 2a. Based on guidance provided by the model, we will systematically investigate the quantification error resulting from complex sample geometry uncertainties when using single and multi-detector configurations. It is found that local surface inclination has the greatest contribution to the quantification error, particularly when using a single X-ray detector.

Finally, the model also provides opportunities to develop new strategies to reduce EDS quantification error due to sample geometry uncertainty. As seen in Figure $2 b$ and $2 c$, the model suggests that grid 
shadowing and an increased detector elevation angle can be used to reduce quantification error. It is anticipated that the flexibility of EDS numerical modeling can continue to aid in reducing uncertainty and can be used to develop improved sample holders optimized for detectors with large collection areas.

\section{References:}

[1] P Lu et al, Nano Letters 16 (2016) p. 2728.

[2] C Parish, Microscopy and Microanalysis, 21 (2015) p. 706.

[3] Z Chen et al, Ultramicroscopy 168 (2016) p. 7.

[4] J Dycus et al, Ultramicroscopy 176 (2016) p. 1.

[5] W Xu et al, Ultramicroscopy 164 (2016) p. 51.

[6] The authors acknowledge the support from Air Force Office of Scientific Research (FA9550-14-10182) and National Science Foundation Graduate Research Fellowship (DGE-1252376). We thank analytical Instrumentation Facility (AIF) at North Carolina State University supported by the State of North Carolina, the National Science Foundation (ECCS-1542015) and the North Carolina Research Triangle Nanotechnology Network from the National Nanotechnology Coordinated Infrastructure.
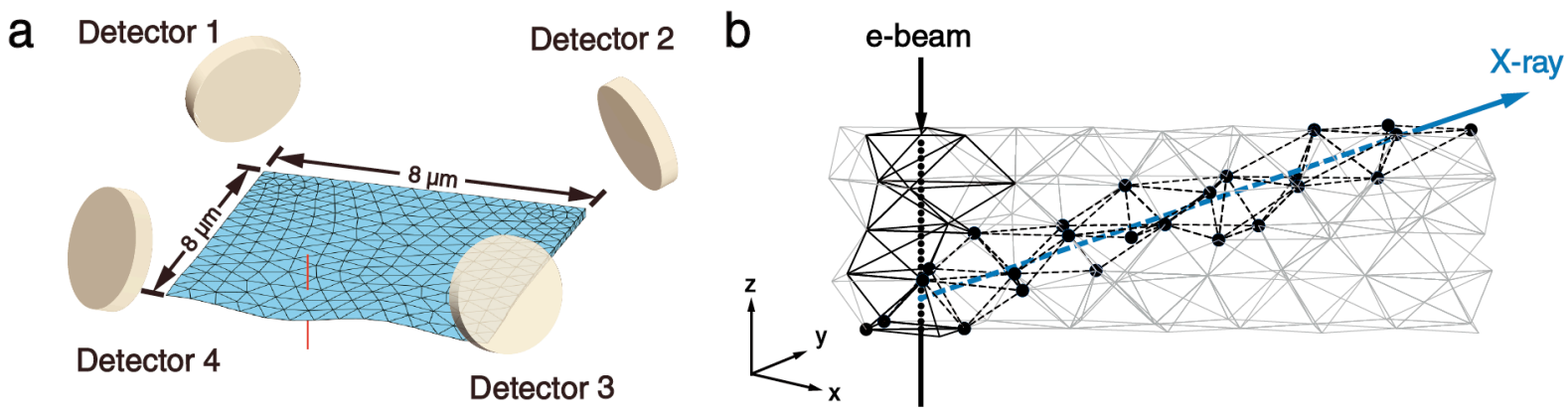

Figure 1. (a) $3 \mathrm{D}$ specimen model with a local distortion near the thin edge. (b) The paths of the incident electron beam and an exiting X-ray path inside the specimen that intersects with the highlighted tetrahedral (in black color).
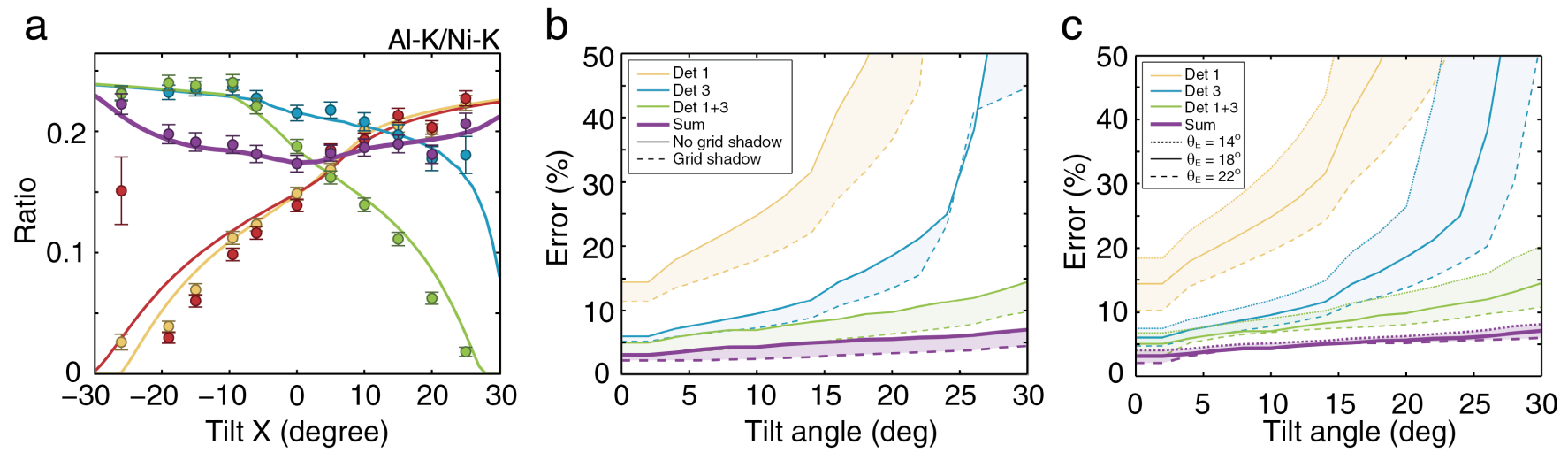

Detector $-1-2-3-4-$ sum

Figure 2. (a) Experiment (dots) and prediction (solid lines) of Al-K/Ni-K ratio as a function of X-tilt, (b) Maximum quantification error as a function of tilt angle $\left( \pm 30^{\circ}\right)$ for individual and multiple detectors configuration with (solid lines) and without grid shadowing (dashed lines). (b) Maximum quantification error for different detector elevation angles. 